\title{
Expression of proinflammatory, proatherogenic genes is reduced by the Mediterranean diet in elderly people
}

\author{
Antonio Camargo ${ }^{1}$, Javier Delgado-Lista ${ }^{1}$, Antonio Garcia-Rios ${ }^{1}$, Cristina Cruz-Teno ${ }^{1}$, \\ Elena M. Yubero-Serrano ${ }^{1}$, Pablo Perez-Martinez ${ }^{1}$, Francisco M. Gutierrez-Mariscal ${ }^{1}$, Pilar Lora-Aguilar ${ }^{1}$, \\ Fernando Rodriguez-Cantalejo ${ }^{2}$, Francisco Fuentes-Jimenez ${ }^{1}$, Francisco Jose Tinahones ${ }^{3}$, \\ Maria M. Malagon ${ }^{4}$, Francisco Perez-Jimenez ${ }^{1} \dagger$ and Jose Lopez-Miranda ${ }^{1}{ }^{1} \dagger$ \\ ${ }^{1}$ Lipids and Atherosclerosis Unit, IMIBIC, Reina Sofía University Hospital, University of Córdoba, CIBER Fisiopatología de la \\ Obesidad y Nutrición (CIBEROBN), Instituto de Salud Carlos III, Córdoba, Spain \\ ${ }^{2}$ Biochemical Laboratory, Hospital Universitario Reina Sofía, Córdoba, Spain \\ ${ }^{3}$ Hospital Virgen de la Victoria, Malaga, CIBEROBN, Instituto de Salud Carlos III, Málaga, Spain \\ ${ }^{4}$ Department of Cell Biology, Physiology and Immunology, University of Córdoba, IMIBIC, CIBEROBN, Spain
}

(Submitted 7 April 2011 - Final revision received 11 July 2011 - Accepted 17 September 2011 - First published online 15 November 2011)

\begin{abstract}
Ageing is an important determinant of atherosclerosis development rate, mainly by the creation of a chronic low-grade inflammation. Diet, and particularly its fat content, modulates the inflammatory response in the fasting and postprandial states. Our aim was to study the effects of dietary fat on the expression of genes related to inflammation (NF- $\mathrm{BB}$, monocyte chemoattractant protein $1(M C P-1)$, TNF- $\alpha$ and $I L-G$ ) and plaque stability (matrix metalloproteinase 9, MMP-9) during the postprandial state of twenty healthy, elderly people who followed three diets for 3 weeks each: (1) Mediterranean diet (Med Diet) enriched in MUFA with virgin olive oil; (2) SFA-rich diet; and (3) lowfat, high-carbohydrate diet enriched in $n$-3 PUFA (CHO-PUFA diet) by a randomised crossover design. At the end of each period, after a 12-h fast, the subjects received a breakfast with a composition similar to the one when the dietary period ended. In the fasting state, the Med Diet consumption induced a lower gene expression of the $p 65$ subunit of NF-кB compared with the SFA-rich diet $(P=0 \cdot 019)$. The ingestion of the Med Diet induced a lower gene postprandial expression of $p 65(P=0 \cdot 033), M C P-1(P=0 \cdot 0229)$ and $M M P-9$ $(P=0 \cdot 041)$ compared with the SFA-rich diet, and a lower gene postprandial expression of $p 65(P=0 \cdot 027)$ and $T N F-\alpha(P=0 \cdot 047)$ compared with the CHO-PUFA diet. Direct plasma quantification mostly reproduced the findings. Our data suggest that consumption of a Med Diet reduces the postprandial inflammatory response in mononuclear cells compared with the SFA-rich and CHO-PUFA diets in elderly people. These findings may be partly responsible for the lower CVD risk found in populations with a high adherence to the Med Diet.
\end{abstract}

Key words: Ageing: Atherosclerosis: Diet: Inflammation

Modern research in CVD indicates that inflammation is the lead underlying the physiopathological mechanism of atherosclerosis $^{(1)}$. The ageing process, on the other hand, promotes atherosclerosis, mainly by an association with chronic low-grade inflammation and increased oxidative stress $^{(2,3)}$. The combination of the above factors allows us to infer that inflammation and ageing are intimately linked in the development of atherosclerosis. Thus, atherosclerosis in aged persons represents a series of highly specific cellular and molecular responses which may be described as an age-related inflammatory disease ${ }^{(4)}$.
In this baseline low-grade inflammation state associated with age, the postprandial state represents a stressful situation of homeostasis by the increase in lipid pro-inflammatory particles, an increase in oxidative stress and a transient increase in pro-inflammatory molecules released by human leucocytes and endothelial cells ${ }^{(5-7)}$.

Peripheral blood mononuclear cells (PBMC) are a subset of leucocytes which include lymphocytes and monocytes, and play a critical role in the immune system. It has been shown that low-grade inflammation is associated with the activation of PBMC and with changes in the expression of

Abbreviations: CHO-PUFA diet, low-fat, high-carbohydrate diet enriched in $n$-3 PUFA; IкB, inhibitors of кB; MCP-1, monocyte chemoattractant protein 1 ; Med Diet, Mediterranean diet; MIF-1, migration inhibitory factor-1; MMP-9, matrix metalloproteinase 9; PBMC, peripheral blood mononuclear cells.

*Corresponding author: Professor J. Lopez-Miranda, fax + 34957 204763, email jlopezmir@uco.es

† These authors contributed equally to this study. 
a variety of cytokines related to inflammation and the immune response, such as $I L-6$ and $T N F-\alpha{ }^{(8,9)}$. PBMC gene expression has been shown to be useful in distinguishing a disease from a healthy state because in the presence of disease, the PBMC alter the normal protein releasing profile to a much more pro-inflammatory profile ${ }^{(10,11)}$. In addition, these cells are being used increasingly for gene expression studies because they can be easily and repeatedly collected in sufficient quantities in contrast to the more invasive sampling of adipose, muscle and liver tissues ${ }^{(8)}$.

Diet, and particularly the fat content of the diet, can modulate the inflammatory and immune responses ${ }^{(12,13)}$. In contrast, there is an age-related increase in the gene expression of some inflammatory molecules (mainly cytokines) ${ }^{(7)}$. However, the maximum evidence of the pro-inflammatory status associated with age is the up-regulation of the expression of $N F-\kappa B$, a key molecule in the regulation of chronic inflammation ${ }^{(4,14)}$.

It has been shown that diet can modulate cytokines and NF- $\mathrm{B}$ expression in the postprandial state in young people. In this population, butter-enriched meals elicit greater postprandial expression of pro-inflammatory cytokines from PBMC compared with the consumption of meals rich in olive oil, the main fat constituent in the Mediterranean diet (Med Diet), or walnuts ${ }^{(15)}$. In addition, the Med Diet reduces NF- $\kappa \mathrm{B}$ activation in PBMC compared with butter- and walnut-enriched diets or Western diets ${ }^{(16,17)}$. Many other studies have shown that the Med Diet reduces the pro-inflammatory response ${ }^{(18-22)}$.

Another factor in atherosclerosis is plaque stability. Atherosclerotic plaques are formed by the accumulation of lipid-rich macrophages, vascular smooth muscle cells, lipids and the extracellular matrix ${ }^{(23-25)}$. Although advanced atherosclerotic lesions can grow sufficiently large to block blood flow, the most important clinical complication is an acute occlusion due to the formation of a thrombus or blood clot, usually associated with plaque rupture, and resulting in coronary events or stroke ${ }^{(26)}$. The major determinants of plaque instability and rupture are progressive lipid accumulation, ongoing inflammation and cap weakening, through which matrix metalloproteinases have been shown to be associated ${ }^{(23)}$.

To date, no intervention studies have thoroughly explored the postprandial effects of diet on inflammation in elderly people and the genes involved in atherosclerotic plaque stability, which is relevant considering that the postprandial state is the condition in which humans spend most of the day. Thus, the aim of the present study was to evaluate the long-term effects of dietary fat on the postprandial expression of pro-inflammatory genes and plaque stability-related genes in PBMC in healthy, elderly people.

\section{Methods \\ Subjects and diets}

The study was performed on twenty free-living elderly subjects (ten men and ten women). Recruitment of the patients at dietary intervention took place between 1 January 2006 and 1 January 2007. Informed consent was obtained from all participants and all underwent a comprehensive medical history, physical examination and clinical chemistry analysis before enrolment. None of the subjects showed evidence of chronic illness, such as hepatic, renal, thyroid or cardiac dysfunction, and they were requested to maintain their regular physical activity and lifestyle and asked to record in a diary any event that could affect the outcome of the study, such as stress, change in smoking habits and alcohol consumption, or intake of foods not included in the experimental design. Of those enrolled, six participants had high blood pressure, two had hyperlipidaemia and three had diabetes mellitus. None of the participants showed evidence of high alcohol consumption or family history of early-onset CVD. None of the participants were active smokers. This study was conducted according to the guidelines laid down in the Declaration of Helsinki and all procedures involving human subjects/patients were approved by The Human Investigation Review Committee at Reina Sofía University Hospital (Córdoba, Spain).

Participants were randomly assigned to receive, in a crossover design, three diets each for a period of 4 weeks: (1) Med Diet enriched in MUFA with virgin olive oil, containing $15 \%$ of energy as protein, $47 \%$ as carbohydrate and $38 \%$ as fat (24\% MUFA (provided by virgin olive oil)), $<10 \%$ SFA, $4 \%$ PUFA of which $0.4 \%$ was $\alpha$-linolenic acid; (2) SFA-rich diet, with $15 \%$ of energy as protein, $47 \%$ as carbohydrate and $38 \%$ as fat (12\% MUFA, $22 \%$ SFA and $4 \%$ PUFA with $0.4 \% \alpha$-linolenic acid); (3) low-fat, high-carbohydrate diet enriched in $n-3$ PUFA (CHO-PUFA diet), with $15 \%$ of energy as protein, $55 \%$ as carbohydrate and $30 \%$ as fat (10\% SFA, $12 \%$ MUFA and $8 \%$ PUFA with $2 \% \alpha$-linolenic acid). The cholesterol intake was constant $(<300 \mathrm{mg} / \mathrm{d})$ throughout the three periods. The $n-3$ PUFA enrichment of the high-CHO and low-fat diet was achieved via the use of natural food components rich in $\alpha$-linolenic acid of plant origin (based on walnuts; Juglans regia L.). Carbohydrate intake from the CHO-PUFA diet was based on the consumption of biscuits, jam and bread. From the Med Diet, $80 \%$ of the MUFA was provided by virgin olive oil, which was used for cooking, salad dressing and as a spread. Butter was used as the main source of SFA during the SFA dietary period.

At the end of each dietary intervention period and after a 12-h fast, the subjects were given a fat meal consisting of a breakfast with a composition similar to that consumed in each of the diets, and with 50-60\% of the subject's daily normal intake of energy. The methodology of the fat-meal test has been validated and extensively used by our unit $^{(15,27,28)}$, and is described next. The composition of the experimental diets was calculated by using the US Department of Agriculture ${ }^{(29)}$ food tables and Spanish food-composition tables for local foodstuffs ${ }^{(30)}$.

Before the start of the intervention period, the volunteers completed a 3-d weighed food diary and an extensive $\mathrm{FFQ}^{(31)}$, which allowed the identification of foods to be modified. Fat foods were administered by dietitians in the intervention study. At the start of the intervention period, each patient was provided with a handbook for the diet to which they had been randomly assigned, which included fourteen menus elaborated with regular solid foods. Advice was given on the foods to choose and those to avoid when eating outside the 
home. At baseline, volunteers were provided with a supply of study foods to last for 2 weeks and collected additional study foods every fortnight or when required. At these times, a 24-h recall of the previous day's food intake and a short food-use questionnaire based on the study foods were completed to monitor and motivate volunteers to adhere to the dietary advice. A score was used to assess the number of food exchanges achieved in the $24-\mathrm{h}$ recall and additional advice was given if either the 24-h recall or the FFQ showed inadequate intake of food-exchange options. Volunteers were asked to complete 3 - $\mathrm{d}$ weighed food diaries at baseline, week 2 and week 4 . Weighed food intake over two weekdays and one weekend day was obtained by using scales provided by the investigators. A dietary analysis software program (Dietsource version 2.0; Novartis, Barcelona, Spain) was used in the nutritional evaluation of the menus. Biochemical laboratory personnel were unaware of the dietary period that each participant was following for each determination.

\section{Sample collection}

Venous blood samples were obtained at the end of each dietary intervention period on fasting state, after a 12-h fast, before breakfast ingestion and at 1, 2 and $4 \mathrm{~h}$ after the ingestion of the breakfast. Samples from the fasting and postprandial states were collected in tubes containing $1 \mathrm{~g}$ EDTA/ 1 and were stored in containers with ice water and kept in the dark. Special care was taken to avoid exposure to air and light; and ambient temperature was maintained. Plasma was separated from whole blood by low-speed centrifugation at $1500 \mathrm{~g}$ for $15 \mathrm{~min}$ at $4^{\circ} \mathrm{C}$ within $1 \mathrm{~h}$ of extraction.

\section{Lipid analysis}

Venous blood samples were collected in tubes containing $1 \mathrm{mg} / \mathrm{ml}$. Plasma was obtained by low-speed centrifugation $(1500 \mathrm{~g})$ for $15 \mathrm{~min}$ at $4^{\circ} \mathrm{C}$ within $1 \mathrm{~h}$ of venepuncture. In order to reduce inter-assay variation, plasma samples were stored at $-80^{\circ} \mathrm{C}$ and analysed at the end of the study. Lipid parameters were assessed with a DDPPII Hitachi modular analyser (Roche, Basel, Switzerland), using specific reagents (Boehringer-Mannheim, Mannheim, Germany). Total plasma cholesterol and TAG concentrations and lipoprotein fractions were measured by colorimetric enzymatic techniques ${ }^{(32,33)}$. HDL-cholesterol levels were measured using colorimetric assay after precipitating the lipoproteins containing apoB with polyethylene glycol $^{(34)}$. LDL-cholesterol concentrations were calculated by using the Friedewald formula based on the cholesterol, TAG and HDL-cholesterol values ${ }^{(35)}$.

\section{Plasma molecules immunoassay}

Plasma concentration of cytokines was determined in duplicate with commercially available ELISA kits: TNF- $\alpha$ (R\&D Systems, Inc., Minneapolis, MN, USA), IL-6 (Bender Med System, Vienna, Austria) and monocyte chemoattractant protein 1 (MCP-1, microbead assays, Bio-Plex, Bio-Rad Laboratories, Inc., Hercules, CA, USA), according to the manufacturers' guidelines.

\section{Isolation of peripheral blood mononuclear cells}

PBMC were isolated within $2 \mathrm{~h}$ after blood was drawn from $30 \mathrm{ml}$ EDTA anticoagulated blood samples. Buffy coats were diluted in a 1:2 ratio in PBS, and the cells were separated in $5 \mathrm{ml}$ Ficoll gradient (lymphocyte isolation solution, Rafer) by centrifugation at $2000 \mathrm{~g}$ for $30 \mathrm{~min}$. PBMC were collected and washed twice with cold PBS. Harvested PBMC were preserved in liquid $\mathrm{N}_{2}$ and stored at $-80^{\circ} \mathrm{C}$ before RNA extraction.

\section{$N F-\kappa B$ activation}

NF- $\mathrm{B}$ (p65) DNA binding activity was determined using the NF- $\mathrm{B}$ (p65) Transcription Factor Assay kit (Cayman Chemical, Ann Arbor, MI, USA). PBMC nuclear protein extracts were obtained following the procedure previously described by Hernández-Presa et al. ${ }^{(36)}$.

\section{RNA extraction and quantitative RT-PCR analysis}

Total RNA was extracted from mononuclear cells with TRI Reagent (Sigma-Aldrich, Inc., St Louis, MO, USA) and purified with RNeasy MiniElute Cleanup Kit (Qiagen, Hilden, Germany). Recovered RNA was quantified using a Nanodrop ND-1000 v3.5.2 spectrophotometer (Nanodrop Technology ${ }^{\circledR}$, Cambridge, UK). RNA integrity was assessed using 1.6\% agarose gel, $1 \times$ TBE (Tris/borate/EDTA). PCR analyses were performed using Mx3005 Thermocycler (Agilent Technologies, La Jolla, CA, USA) and iQ SYBR ${ }^{\circledR}$ Green Supermix (Bio-Rad Laboratories, Inc.) commercial kits in a final volume of $20 \mu \mathrm{l}$ with $10 \mathrm{pmol}$ of each primer. Each reaction was performed on $1 \mu \mathrm{l}$ of $1: 5(\mathrm{v} / \mathrm{v})$ dilution of the first complementary DNA strand, synthesised using $1 \mu \mathrm{g}$ of total RNA by the commercial kit iScript ${ }^{\circledR}$ CDNA Synthesis Kit (Bio-Rad Laboratories, Inc.) according to the manufacturer's instructions. The reaction was incubated at $96^{\circ} \mathrm{C}$ for $3 \mathrm{~min}$, followed by forty cycles of $30 \mathrm{~s}$ at $96^{\circ} \mathrm{C}, 30 \mathrm{~s}$ at $62^{\circ} \mathrm{C}, 20 \mathrm{~s}$ at $72^{\circ} \mathrm{C}$ and $10 \mathrm{~s}$ at $80^{\circ} \mathrm{C}$ where fluorescence was measured to avoid primer-dimer and background signals. Primers used were: p65-Up (5'-CCCTGTCCTGATGGTCAGCTCCCT-3'), p65-Low (5'-CTCAAACGCTGGTGTTAGGCACAGGG-3 $\left.{ }^{\prime}\right)$, ІкB $\alpha$-Up $\quad\left(5^{\prime}\right.$ CACTCCATCCTGAAGGCTACCAAC- $3^{\prime}$ ), ІкB $\alpha$-Low (5'-CACACTTCAACAGGAGTGACACCAG- $\left.3^{\prime}\right)$, MCP1-Up (5'-CATGAAAGTCTCTGCCGCCCT-3'), MCP1-LOW (5'-CACTTGCTGCTGGTGATTCTTCTAT- $\left.3^{\prime}\right)$, TNF $\alpha$-Up ( $5^{\prime}$-AAGAGTTCCCCAGGGACCTCT- $3^{\prime}$ ), TNF $\alpha$-Low ( $5^{\prime}$-CCTGGGAGTAGATGAGGTACA- $3^{\prime}$ ), MIF1-Up (5'-CTCTCCGAGCTCACCCAGCAG-3 $\left.{ }^{\prime}\right)$, MIF1-Low (5'-CGCGTTCATGTCGTAATAGTT-3'), MMP-9-Up (5'-CCCATTTCGACGATGACGAGTTGTG-3'), MMP-9-Low (5'-GGAGTAGGATTGGCCTTGGAAGATG-3'), IL6-Up (5'-CACCTCTTCAGAACGAATTGACAAAC-3'), IL6-LOW (5'-CTCATTGAATCCAGATTGGAAGC-3'), RPL13-Up (5'-CCTGGAGGAGAAGAGGAAAGAGA-3') and RPL13-Low (5'-TTGAGGACCTCTGTGTATTTGTCAA- $\left.3^{\prime}\right)$. Specificity of PCR amplifications was verified by a melting curve program $\left(60-95^{\circ} \mathrm{C}\right.$, with a heating rate of $0.5^{\circ} \mathrm{C} / \mathrm{s}$ and a continuous fluorescence measurement) and analysed by electrophoresis on a $1.6 \%$ agarose gel, TBE $1 \times$. Expression values were obtained as relative expression 
of the target gene $v$, the constitutively expressed RPL13a (ribosomal protein L13a) gene (relative expression $=$ $\left.2^{-\left(C_{\mathrm{t}} \text {,Target gene }-C_{\mathrm{t}}, \text { Reference gene }\right)}\right)$.

\section{Statistical analysis}

SPSS statistical software, version 15.0 (SPSS, Inc., Chicago, IL, USA) was used for statistical comparisons. The KolmogorovSmirnov test was used to check the normality of the distribution of variance values. When the data did not show the normality of the distribution, we log-transformed them to obtain a normal distribution. In order to evaluate data variation, an ANOVA for repeated measures was performed, followed by Bonferroni's correction for multiple comparisons. The contrast statistic used was the sphericity assumption. The contrast statistic used when the sphericity assumption was not satisfied was Greenhouse-Geisser. Post hoc statistical analysis was completed to identify significant differences between dietary treatments. A probability value of less than 0.05 was considered significant. A study of the relationship among the parameters was carried out using Pearson's linear correlation coefficient. A probability value of less than 0.05 was considered significant. All data presented in the text and tables are expressed as means and standard errors.

\section{Results}

\section{Baseline characteristics of the participants}

The baseline characteristics of the twenty healthy participants who completed the three dietary intervention periods have been described by Marin et al. ${ }^{(37)}$. Briefly, subjects (ten men and ten women) had an average age of 67.1 (SEM 4.52) years, and an average BMI of $31.9(\operatorname{sem} 5.50) \mathrm{kg} / \mathrm{m}^{2}$. The subjects were non-smokers and none of the subjects had any evidence of CVD.

\section{Dietary intake and inflammation regulatory genes}

We studied the effect of the long-term ingestion of the three different diets on NF- $\mathrm{B}$ activation, but no significant differences between the diets were found. In addition, we studied the expression of the $p 65$ gene, a subunit of the NF-кB transcription factor, and the $I \kappa B \alpha$ gene, a member of the IкB family (inhibitors of $\kappa \mathrm{B}$ ), which is involved in NF- $\kappa \mathrm{B}$ inactivation by sequestration in the cytoplasm (Fig. 1).

In the fasting state, we found that consumption of the Med Diet induced lower $N F-\kappa B p 65$ gene expression compared with consumption of an SFA-rich diet $(P=0 \cdot 019)$. In addition, we observed that the ingestion of an SFA-rich diet induced higher postprandial expression of the $N F-\kappa B p 65$ gene than the Med Diet $(P=0.006)$ or the CHO-PUFA diet $(P=0.031)$ throughout the whole postprandial period. Furthermore, $2 \mathrm{~h}$ after the intake of a Med Diet breakfast, we found a statistically significant lower postprandial expression of the $p 65$ gene than after the SFA-rich $(P=0.033)$ and CHO-PUFA breakfast $(P=0 \cdot 027)$. Consumption of the Med Diet induced a higher postprandial expression of $I \kappa B \alpha$ throughout the whole postprandial period $(P=0.043)$ as compared to the other diets.
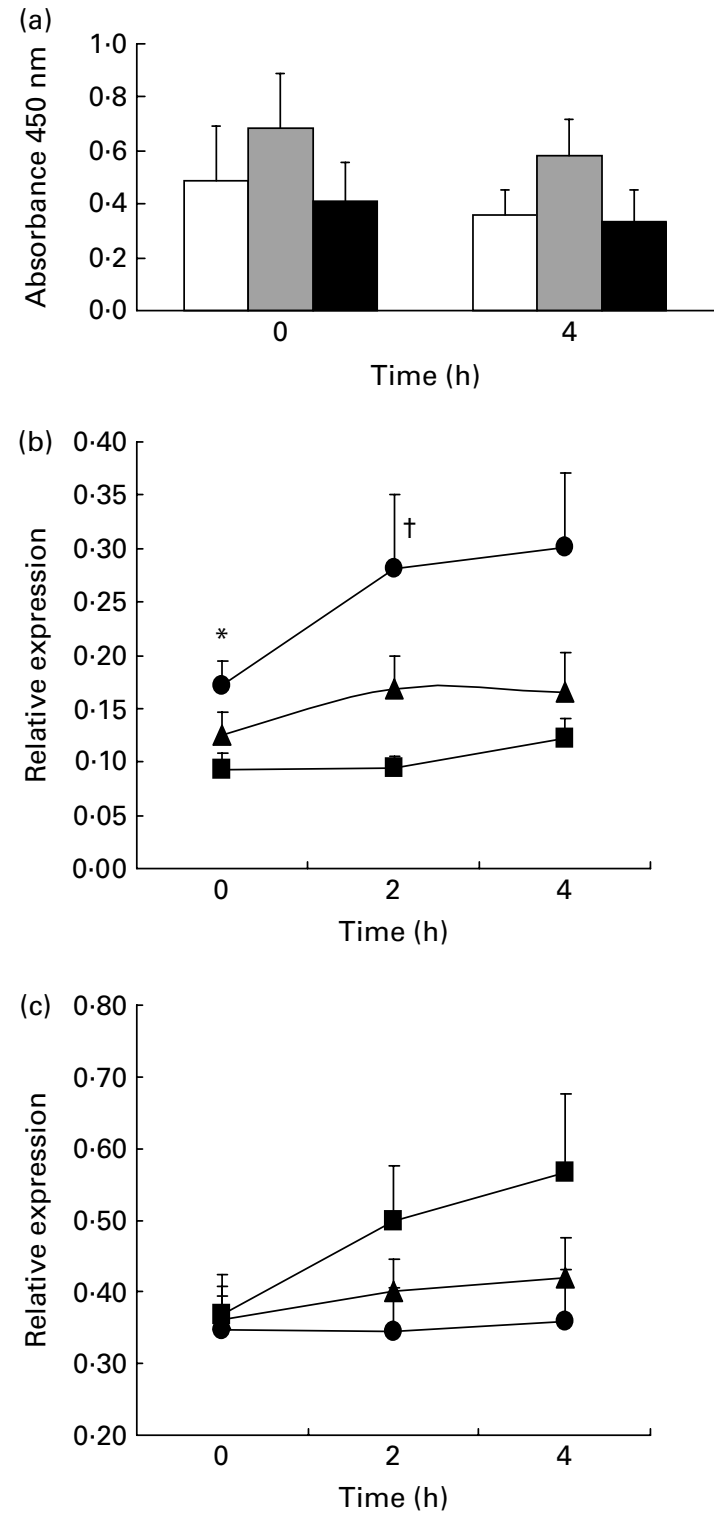

Fig. 1. NF-кB activation and expression of regulatory genes. ANOVA for repeated measurements: $P 1$, effect of diet; $P 2$, time effect; $P 3$, diet $\times$ time interaction. Values are means, with their standard errors represented by vertical bars of (a) NF-kB activation ( $n$ 13; $P 1=0.274, P 2=0.206, P 3=0.786$; Mediterranean diet (Med Diet, $\square$ ), SFA-rich diet $(\square)$, low-fat, high-carbohydrate diet enriched with $n$-3 PUFA (CHO-PUFA diet, $\mathbf{\square})$ ), (b) $p 65$ $(N F-\kappa B ; P 1=0.002, P 2=0.045, P 3=0.382$; Med Diet $(\square)$, SFA-rich diet $(\bullet)$, CHO-PUFA diet $(\boldsymbol{\Delta}))$ and (c) $I_{\kappa} B \alpha(P 1=0.043, P 2=0.137, P 3=0.280$; Med Diet $(\boldsymbol{\square})$, SFA-rich diet $(\bullet)$, CHO-PUFA diet $(\boldsymbol{\Delta})$ ) relative expression $(n \mathbf{2 0})$ in peripheral blood mononuclear cells. *Mean values were significantly different for SFA-rich diet from those of Med Diet $(P<0.05)$. †Mean values were significantly different for SFA-rich diet from those of Med Diet and CHOPUFA diet $(P<0.05)$.

\section{Dietary intake and pro-inflammatory cytokines}

To determine whether the intake of the three diets could regulate the postprandial expression of different pro-inflammatory cytokines in PBMC, we studied the gene expression for $M C P-1, T N F-\alpha$ and $I L-6$ (Fig. 2).

No statistically significant differences in the expression of these genes were detected during the fasting state between 

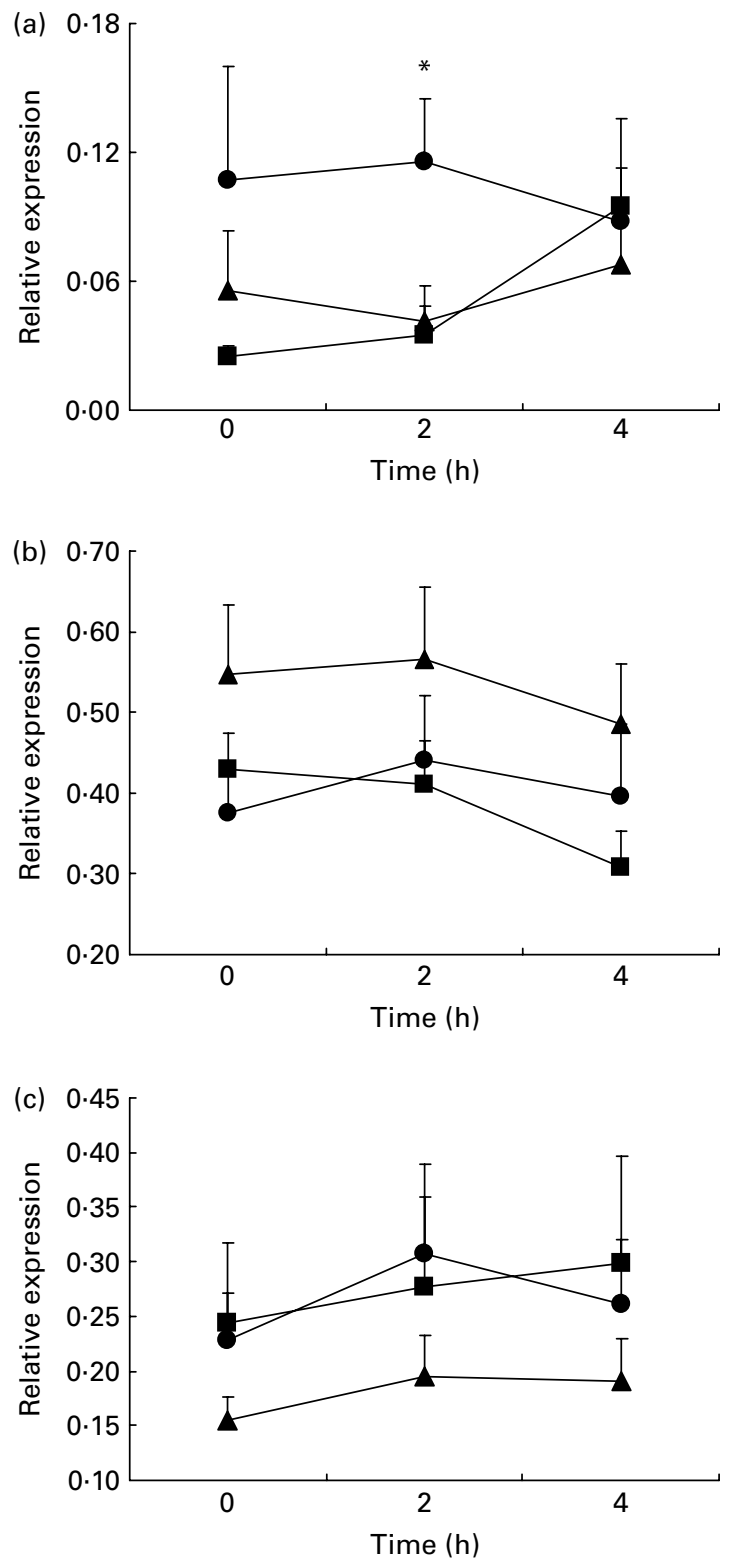

Fig. 2. Postprandial cytokine gene expression. Values are means, with their standard errors represented by vertical bars of relative expression in peripheral blood mononuclear cells. Monocyte chemoattractant protein 1 (MCP-1) gene expression values were log-transformed to obtain a normal distribution. ANOVA for repeated measurements ( $n$ 20): $P 1$, effect of diet; $P 2$, time effect; $P 3$, diet $\times$ time interaction. (a) $M C P-1(P 1=0.017, P 2=0.150, P 3=0.199)$, (b) $T N F-\alpha(P 1=0.028, P 2=0.178, P 3=0.840)$ and (c) $I L-6 \quad(P 1=0.246$, $P 2=0.307, P 3=0.898$ ). Mediterranean diet (Med Diet, $\square$ ); SFA-rich diet $(\bullet)$ low-fat, high-carbohydrate diet enriched with $n$-3 PUFA (CHO-PUFA diet, $\mathbf{\Lambda}$ ). ${ }^{*}$ Mean values were significantly different for SFA-rich diet $v$. Med Diet $(P<0.05)$.

diets. However, we observed that the ingestion of an SFA-rich diet induced a higher expression of the $M C P-1$ gene than the Med Diet $(P=0.022)$ when assessing the postprandial measurements together $(0,2$ and $4 \mathrm{~h})$. In the post hoc tests, these differences were significant at $2 \mathrm{~h}(P=0.018)$. When we studied the expression of the $T N F-\alpha$ gene, we showed that the ingestion of the CHO-PUFA diet induced a higher
$T N F-\alpha$ postprandial gene expression than the Med Diet $(P=0.047)$. We also studied the expression of the $I L-6$ gene, but no significant differences were found between the three diets.

In addition, we also analysed the plasma levels of MCP-1, TNF- $\alpha$ and IL-6 (Fig. 3). Consumption of an SFA-rich diet induced a postprandial increase in the MCP-1 plasma concentration $(P=0.015)$. The TNF- $\alpha$ plasma level decreased $(P=0.008)$ and the IL-6 increased $(P<0 \cdot 001)$ in the postprandial state, irrespective of the diet consumed.
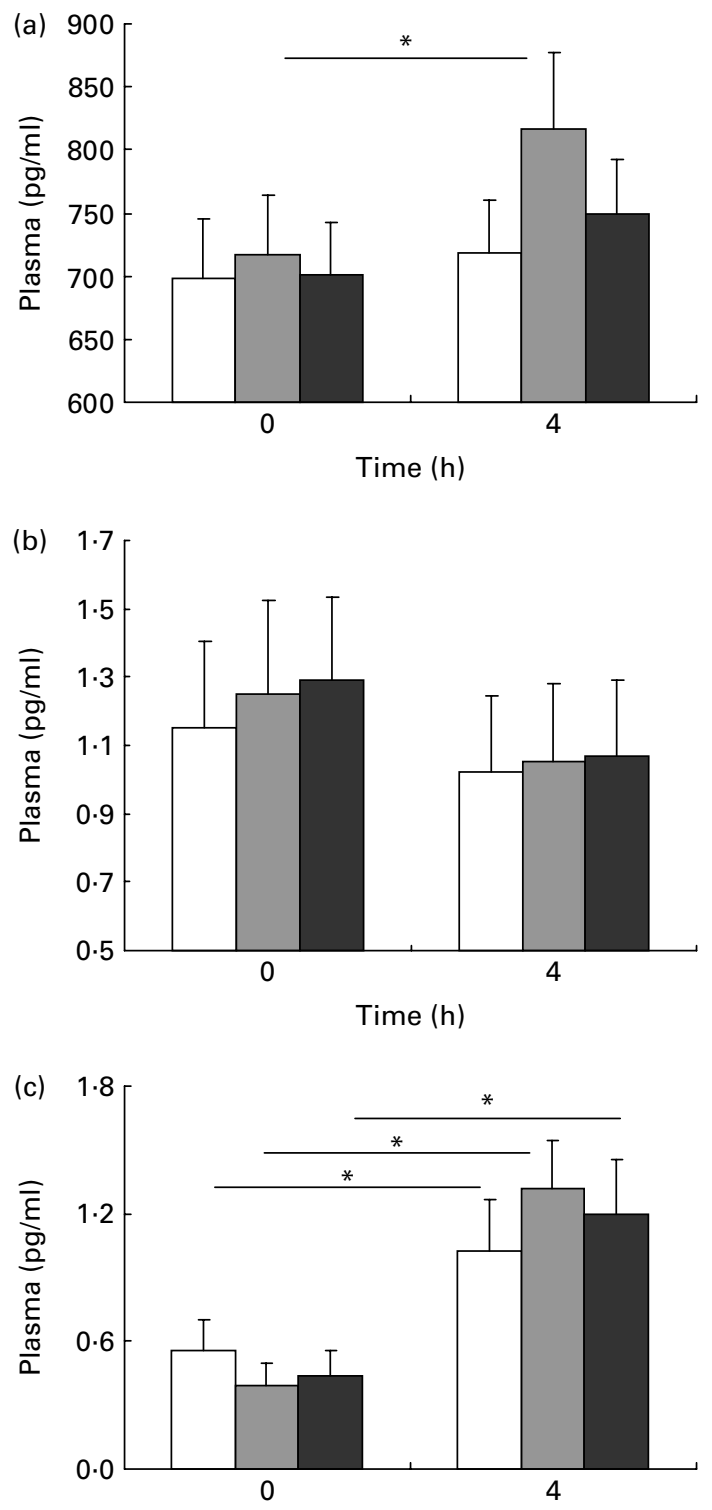

Time (h)

Fig. 3. Monocyte chemoattractant protein 1, TNF- $\alpha$ and IL-6 plasma levels. ANOVA for repeated measurements $(n 20)$ : $P 1$, effect of diet; $P 2$, time effect; $P 3$, diet $\times$ time interaction. (a) Monocyte chemoattractant protein 1 $(P 1=0.293 ; \quad P 2=0.026 ; \quad P 3=0.266), \quad(b) T N F-\alpha \quad(P 1=0.723 ; \quad P 2=0.008$; $P 3=0.817)$ and (c) IL-6 $(P 1=0.933 ; P 2<0.001 ; P 3=0.176)$ plasma levels. Values are means, with their standard errors represented by vertical bars of plasma levels. Mediterranean diet (Med Diet, $\square$ ); SFA-rich diet ( $\square$ ); low-fat, high-carbohydrate diet enriched with $n$-3 PUFA (CHO-PUFA diet, $\square$ ). * Mean values were significantly different $(P<0.05)$. 
Table 1. Gene expression of atherosclerotic plaque stability-related genes* (Mean values with their standard errors)

\begin{tabular}{|c|c|c|c|c|}
\hline \multirow[b]{2}{*}{ Time and diet } & \multicolumn{2}{|c|}{$M I F-1$} & \multicolumn{2}{|c|}{ MMP-9 } \\
\hline & Mean & SEM & Mean & SEM \\
\hline \multicolumn{5}{|l|}{$\mathrm{Oh}$} \\
\hline Med Diet & 0.104 & $0 \cdot 013$ & $0 \cdot 124$ & 0.022 \\
\hline SFA-rich diet & 0.133 & 0.012 & 0.404 & 0.207 \\
\hline CHO-PUFA diet & 0.139 & 0.009 & 0.557 & $0 \cdot 266$ \\
\hline \multicolumn{5}{|l|}{$2 \mathrm{~h}$} \\
\hline Med Diet & 0.116 & 0.011 & 0.362 & 0.155 \\
\hline SFA-rich diet & 0.134 & 0.016 & 0.768 & 0.278 \\
\hline CHO-PUFA diet & 0.123 & 0.011 & 0.799 & 0.377 \\
\hline \multicolumn{5}{|l|}{$4 \mathrm{~h}$} \\
\hline Med Diet & 0.112 & $0 \cdot 011$ & 0.396 & 0.164 \\
\hline SFA-rich diet & 0.117 & 0.015 & 0.595 & 0.279 \\
\hline CHO-PUFA diet & 0.137 & 0.013 & 0.623 & 0.295 \\
\hline \multicolumn{5}{|c|}{ Global analysis $P$ values } \\
\hline Time effect & \multicolumn{2}{|c|}{0.243} & \multicolumn{2}{|c|}{0.047} \\
\hline Diet effect & \multicolumn{2}{|c|}{0.883} & \multicolumn{2}{|c|}{0.021} \\
\hline Diet by time effect & \multicolumn{2}{|c|}{0.304} & \multicolumn{2}{|c|}{0.273} \\
\hline
\end{tabular}

MIF-1, migration inhibitory factor-1; MMP-9, matrix metalloproteinase 9; Med Diet Mediterranean Diet; CHO-PUFA diet, low-fat, high-carbohydrate diet enriched in $n$-3 PUFA.

* Mean values with their standard errors of relative expression in peripheral blood mononuclear cells. MMP-9 gene expression values were log-transformed to obtain a normal distribution. ANOVA for repeated measures $(n 20)$.

\section{Dietary intake and atherosclerotic plaque stability}

To evaluate whether the ingestion of three different diets could affect atherosclerotic plaques, we studied the expression of matrix metalloproteinase 9 ( $M M P-9)$, a metalloproteinase shown to contribute to plaque instability, and macrophage migration inhibitory factor-1 $(M I F-1)$, one of the stimulators of MMP-9 (Table 1). Although no statistically significant differences between the diets were found on $M I F-1$ or $M M P-9$ gene expression in the fasting state, the ingestion of an SFA-rich diet induced a higher postprandial $M M P-9$ gene expression than the Med Diet $(P=0.041)$. We did not find any differences in the gene expression of MIF-1 depending on the diets.

\section{Correlation between pro-inflammatory cytokine level and lipid parameters}

We studied the relationship between the expression of inflammatory genes and lipid parameters. Postprandial lipaemia for this population under study has been described by Marin et $a l .{ }^{(37)}$. The plasma total cholesterol concentration positively correlated with the $N F-\kappa B$ p65 sub-unit gene expression ( $R 0.260, P=0.044$ ) in the fasting state. In addition, both the total cholesterol and LDL-cholesterol plasma concentration positively correlated with the $M I F-1$ gene expression ( $R$ 0.257, $P=0.047$; and $R 0.257, P=0.049$, respectively) in the postprandial state ( $4 \mathrm{~h}$ after breakfast). Interestingly, the plasma level of MCP-1 and the HDL-cholesterol plasma concentration were negatively correlated in the fasting and postprandial states ( $4 \mathrm{~h}$ after breakfast $(R-0.257, P=0.047$; and $R-0.335, P=0.009$, respectively)).

\section{Discussion}

In the present study, we observed a lower expression of several inflammatory genes after the consumption of a Med Diet compared with SFA-rich ( $p 65$ and $M C P-1$ ) and CHO-PUFA diets $(p 65$ and $T N F-\alpha$ ), and an increase in the expression of the anti-inflammatory gene, $I \kappa B \alpha$, in elderly people during the postprandial state. Additionally, the gene expression of $M M P-9$, a marker of plaque instability, was lower after consumption of a Med Diet compared with an SFA-rich diet during the postprandial state.

Recent pathological evidence indicates that major chronic age-related diseases, such as atherosclerosis, arthritis, dementia, osteoporosis and CVD, are linked to a state of increased inflammation $^{(7,38)}$. The $\mathrm{NF}-\kappa \mathrm{B}$ transcription factor and its target genes, which include several pro-inflammatory cytokines, are key factors in the regulation of inflammation. In summary, the NF- $\mathrm{B}$ transcription factor acts as a 'trigger', the activation of which leads to a cascading expression of genes and proteins, which leads to inflammatory activation. NF-кB is involved, among other molecules, in the pathogenesis of atherosclerosis ${ }^{(39)}$. The activation of this factor may be, among others, regulated by dietary fat ${ }^{(16)}$. Our group has already demonstrated that the Med Diet decreases NF- $\mathrm{\kappa B}$ activation in PBMC when compared with butter- and walnutenriched diets or a typical Western $\operatorname{diet}^{(16,17)}$ in healthy young people. This factor is constitutively formed by two sub-units (p65 and p50), the activation of which leads to the split from IкB and migration to the nucleus. ІкB stabilises the NF- $\kappa \mathrm{B}$ molecule in the cytoplasm, maintaining it in an 'unactivated' state. Only the release of this third sub-unit allows p65 and p50 to migrate to the nucleus to initiate the pro-inflammatory effects of $\mathrm{NF}-\kappa \mathrm{B}^{(40)}$. In the present study, we observed an increase in the expression of the inhibitory $I \kappa B \alpha$ sub-unit and a decrease in the activating $p 65$ sub-unit after the Med Diet, especially in comparison to the SFA-rich, but also in comparison to the low-fat diet. These combined effects show the underlying mechanisms that cause the lower NF-кB activation reported after the Med Diet, and demonstrate that this lower activation is induced by a double mechanism as follows: I $B \alpha$ gene expression is increased, while the pro-activator p 65 sub-unit is reduced.

We administered a Med Diet enriched in MUFA with virgin olive oil. In addition to the benefits conferred by its lipid content, virgin olive oil has other biological effects as it contains hundreds of non-fat components with great biological potential, including vitamin E, carotenes, squalene, flavonoids, phenolic compounds and others with antioxidant properties ${ }^{(41-43)}$, which could be responsible for a lower postprandial inflammatory response by reducing the pro-oxidative state that activates the genes implicated in the inflammatory response during the postprandial period $^{(44)}$

Additionally, minor components of olive oil, such as phenolic compounds, which have also been shown to exert antiinflammatory properties ${ }^{(41-43)}$, could be responsible for at least some of the observed effects on NF- $\mathrm{KB}$ shown in in vitro studies ${ }^{(45,46)}$. 
However, one of the limitations of this study was deducing whether the observed effects after Med Diet consumption are due to MUFA content, minor components of olive oil, or a combination of both.

In contrast to the report from the PREDIMED (Prevención con Dieta Mediterránea) study, which showed the effects of the Med Diet on plasma inflammatory markers ${ }^{(47)}$ and expression of inflammatory genes on $\mathrm{PBMC}^{(48)}$, our study did not show significant differences between the diets during the fasting state on the inflammatory markers analysed.

Nevertheless, our study showed that consumption of the Med Diet induced a lower expression of the $M C P-1$ gene than an SFA-rich diet when assessing the postprandial measurements together. MCP-1 (also known as small inducible cytokine A2) and monocyte chemotactic and activating factor play a fundamental role in the recruitment of monocytes to sites of injury and infection, and have been shown to increase in clinical models of chronic inflammation, such as rheumatoid arthritis or lupus. Furthermore, MCP-1 plays a critical role in atherosclerotic plaque formation, by initiating fatty streak formation ${ }^{(49)}$. In addition, in the present study, the Med Diet was associated with a lower expression of the $T N F-\alpha$ gene, which acts as an activator of a cascade of cytokine production ${ }^{(50)}$, compared with the consumption of a CHO-PUFA diet.

Along with the progression of atherosclerosis, rupture of the atherosclerotic plaque is fundamental to the appearance of cardiovascular events. Furthermore, this rupture of the plaque and the subsequent inflammatory and pro-aggregant responses is the key mechanism responsible for these clinical events $^{(51,52)}$. In our study, the postprandial expression of MMP-9, a metalloprotease with an important role in the unstabilisation of the plaque, was lower after consumption of the Med Diet. This effect of the consumption of the Med Diet could be due to the olive oil phenolic compound, as in vitro studies have shown that these compounds prevent the stimulation of MMP-9 expression and secretion in TNF$\alpha$-treated THP-1 (human acute monocytic leukaemia cell line) cells ${ }^{(45)}$ by impairing NF-кB signalling. Thus, consumption of a Med Diet may not only influence the rate by which atherosclerosis develops, but also promotes the stability of the plaque ${ }^{(48)}$ by reducing $M M P-9$ gene expression, possibly by the inhibition of metalloproteinase actions performed by olive oil phenolic compounds as suggested by Hashim et al. ${ }^{(53)}$, which has been shown in green tea polyphenols ${ }^{(54,55)}$.

When analysing our results, we should keep in mind that we performed the study during the postprandial state (not the fasting state), which is the most common state in the populations of Western countries. The postprandial state following a fatty meal induces a 'physiological' inflammatory response ${ }^{(12,13)}$, which may have allowed us to discover the differences in gene expression of the PBMC, depending on the type of diet. Thus, part of the differences in the gene expression of the PBMC may be due to the pro-inflammatory state, which is why the genes could not be detected in the fasting state. To confirm the pro-inflammatory state in which the PBMC were extracted, other pro-inflammatory markers (MCP-1 and IL-6 plasma levels) were augmented, regardless of the diet. Interestingly, gene expression of these proteins was not increased; proteins increased in the blood, but the gene expression in PBMC did not rise, which may indicate that the increased level in the postprandial state may exceed the regulation of PBMC. Other additional potential causes for this apparent lack of correlation between IL-6 plasma levels and mononuclear gene expression may be that the synthesis and secretion processes of these proteins do not occur simultaneously ${ }^{(56,57)}$ and/or that there are additional regulatory mechanisms in the secretory pathways ${ }^{(58)}$

It has been suggested that ageing may contribute to changes in immune system activity, resulting in altered cell surface marker expression and cytokine levels in the elderly population compared with young people ${ }^{(59)}$. Our findings show that this age-related increase in inflammation may be, at least partially during the postprandial state, modulated by diet.

In conclusion, our data suggest that consumption of the Med Diet reduces the expression of inflammatory genes in PBMC compared with the SFA-rich and CHO-PUFA diets. Additionally, the Med Diet reduced the expression of metalloproteinases in relation to atherosclerotic plaque instability compared with an SFA-rich diet during the postprandial state. These findings may be partly responsible for the lower CVD risk in populations with a high adherence to the Med Diet.

\section{Acknowledgements}

The CIBER Fisiopatología de la Obesidad y Nutrición is an initiative of the Instituto de Salud Carlos III, Madrid, Spain. This study was supported in part by research grants from the Spanish Ministry of Science and Innovation (AGL 200407907, AGL2006-01979 and AGL2009-12270 to J. L.-M., SAF07-62005 to F. P.-J. and FIS PI10/01041 to P. P.-M., PI10/ 02412 to F. P.-J.); Consejería de Economía, Innovación y Ciencia, Proyectos de Investigación de Excelencia, Junta de Andalucía (P06-CTS-01425 to J. L.-M., CTS-03039 to M. M. M., CTS5015 and AGR922 to F. P.-J.); Consejería de Salud, Junta de Andalucía (06/128, 07/43, and PI0193/09 to J. L.-M., 06/129 to F. P.-J., 0118/08 to F. F.-J., PI-0252/09 to J. D.-L. and PI-0058/10 to P. P.-M.); Fondo Europeo de Desarrollo Regional. We thank $\mathrm{M}^{\mathrm{a}}$ Jose Gomez-Luna for technical support. The contributions of the authors to the study were as follows: A. C., J. D.-L., J. L.M. and F. P.-J. designed the research; A. C., J. D.-L. and P. P.-M. contributed to the provision of study materials and subjects; A. C., C. C.-T., E. Y.-S., F. G.-M., F. R.-C. and P. L.-A. performed the research; F. R.-C. contributed new reagents/analytical tools; A. C., F. G.-M. and P. L.-A. were responsible for the collection and assembly of data; A. C., J. D.-L., A. G.-R., C. C.-T., E. Y.-S. and P. P.-M. analysed the data; J. D.-L., P. P.-M., J. L.-M. and F. P.-J. provided statistical expertise; A. C. wrote the paper; F. F.-J., F. J. T., M. M. M., F. P.-J. and J. L.-M. were responsible for critical review of the manuscript; P. P.-M., J. D.-L., M. M. M., F. P.-J. and J. L.-M. obtained funding for the present study. None of the authors has any conflict of interests that could affect the performance of the work or the interpretation of the data. 


\section{References}

1. Kriszbacher I, Koppan M \& Bodis J (2005) Inflammation, atherosclerosis, and coronary artery disease. $N$ Engl J Med 353, 429-430.

2. Herrera MD, Mingorance C, Rodriguez-Rodriguez R, et al. (2009) Endothelial dysfunction and aging: an update. Ageing Res Rev 9, 142-152.

3. Bruunsgaard H, Pedersen M \& Pedersen BK (2001) Aging and proinflammatory cytokines. Curr Opin Hematol 8, 131-136.

4. Ross R (1999) Atherosclerosis - an inflammatory disease. $N$ Engl J Med 340, 115-126.

5. Parks EF (2001) Recent findings in the study of postprandial lipemia. Curr Atheroscler Rep 3, 462-470.

6. van Oostrom AJ, Rabelink TJ, Verseyden C, et al. (2004) Activation of leukocytes by postprandial lipemia in healthy volunteers. Atherosclerosis 177, 175-182.

7. Chung HY, Sung B, Jung KJ, et al. (2006) The molecular inflammatory process in aging. Antioxid Redox Signal 8, $572-581$.

8. de Mello VD, Kolehmainen M, Schwab U, et al. (2008) Effect of weight loss on cytokine messenger RNA expression in peripheral blood mononuclear cells of obese subjects with the metabolic syndrome. Metabolism 57, 192-199.

9. Ghanim H, Aljada A, Hofmeyer D, et al. (2004) Circulating mononuclear cells in the obese are in a proinflammatory state. Circulation 110, 1564-1571.

10. Maas K, Chan S, Parker J, et al. (2002) Cutting edge: molecular portrait of human autoimmune disease. J Immunol 169, 5-9.

11. Burczynski ME \& Dorner AJ (2006) Transcriptional profiling of peripheral blood cells in clinical pharmacogenomic studies. Pharmacogenomics 7, 187-202.

12. Paschos GK, Rallidis LS, Liakos GK, et al. (2004) Background diet influences the anti-inflammatory effect of alphalinolenic acid in dyslipidaemic subjects. Br J Nutr 92, 649-655.

13. Baer DJ, Judd JT, Clevidence BA, et al. (2004) Dietary fatty acids affect plasma markers of inflammation in healthy men fed controlled diets: a randomized crossover study. Am J Clin Nutr 79, 969-973.

14. Lawrence $\mathrm{T}$ (2009) The nuclear factor NF-kappaB pathway in inflammation. Cold Spring Harb Perspect Biol 1, a001651.

15. Jiménez-Gómez Y, López-Miranda J, Blanco-Colio LM, et al. (2008) Olive oil and walnut breakfasts reduce the postprandial inflammatory response in mononuclear cells compared with a butter breakfast in healthy men. Atherosclerosis 204, e70-e76.

16. Perez-Martinez P, Lopez-Miranda J, Blanco-Colio L, et al. (2007) The chronic intake of a Mediterranean diet enriched in virgin olive oil, decreases nuclear transcription factor kappaB activation in peripheral blood mononuclear cells from healthy men. Atherosclerosis 194, 141-146.

17. Bellido C, López-Miranda J, Blanco-Colio LM, et al. (2004) Butter and walnuts, but not olive oil, elicit postprandial activation of nuclear transcription factor kappaB in peripheral blood mononuclear cells from healthy men. Am J Clin Nutr 80, 1487-1491.

18. Esposito K, Marfella R, Ciotola M, et al. (2004) Effect of a Mediterranean-style diet on endothelial dysfunction and markers of vascular inflammation in the metabolic syndrome: a randomized trial. JAMA 292, 1440-1446.

19. Meydani M (2005) A Mediterranean-style diet and metabolic syndrome. Nutr Rev 63, 312-314.
20. Estruch R, Martínez-González MA, Corella D, et al. (2006) Effects of a Mediterranean-style diet on cardiovascular risk factors: a randomized trial. Ann Intern Med 145, 1-11.

21. Fuentes F, López-Miranda J, Pérez-Martínez P, et al. (2008) Chronic effects of a high-fat diet enriched with virgin olive oil and a low-fat diet enriched with alpha-linolenic acid on postprandial endothelial function in healthy men. Br J Nutr 100, 159-165.

22. López-Miranda J, Pérez-Jiménez F, Ros E, et al. (2010) Olive oil and health: summary of the II international conference on olive oil and health consensus report, Jaén and Córdoba (Spain) 2008. Nutr Metab Cardiovasc Dis 20, 284-294.

23. Kong YZ, Yu X, Tang JJ, et al. (2005) Macrophage migration inhibitory factor induces MMP-9 expression: implications for destabilization of human atherosclerotic plaques. Atherosclerosis 178, 207-215.

24. Speidl WS, Kastl SP, Hutter R, et al. (2011) The complement component $\mathrm{C} 5 \mathrm{a}$ is present in human coronary lesions in vivo and induces the expression of MMP-1 and MMP-9 in human macrophages in vitro. FASEB J 25, 35-44.

25. Santos-Gallego CG, Bayón J \& Badimón JJ (2010) Thrombi of different pathologies: implications for diagnosis and treatment. Curr Treat Options Cardiovasc Med 12, 274-291.

26. Lusis AJ (2000) Atherosclerosis. Nature 407, 233-241.

27. Jiménez-Gómez Y, Marín C, Pérez-Martínez P, et al. (2010) A low-fat, high-complex carbohydrate diet supplemented with long-chain $(n-3)$ fatty acids alters the postprandial lipoprotein profile in patients with metabolic syndrome. $J$ Nutr 140, 1595-1601.

28. Lopez-Miranda J, Williams C \& Lairon D (2007) Dietary, physiological, genetic and pathological influences on postprandial lipid metabolism. Br J Nutr 98, 458-473.

29. Human Nutrition Information Service (1987) Agriculture Handbook No. 8. Department of Agriculture Composition of Foods. Washington, DC: US Government Printing Office.

30. Varela G (1980) Tablas de composición de alimentos (Spanish) (Food Composition Tables). Madrid: Instituto de Nutrición, CSIC.

31. Martin-Moreno JM, Boyle P, Gorgojo L, et al. (1993) Development and validation of a food frequency questionnaire in Spain. Int J Epidemiol 22, 512-519.

32. Bucolo G \& David H (1973) Quantitative determination of serum triglycerides by use of enzymes. Clin Chem 19, 476-482.

33. Allain CC, Poon LS, Chang CSG, et al. (1974) Enzymatic determination of total serum cholesterol. Clin Chem 20 , 470-475.

34. Brigg CJ, Anderson D, Johnson P, et al. (1981) Evaluation of the polyethylene glycol precipitation method for the estimation of high-density lipoprotein cholesterol. Ann Clin Biochem 18, 177-181.

35. Friedewald WT, Levy RI \& Fredrickson DS (1972) Estimation of the concentration of low-density lipoprotein cholesterol in plasma without use of a preparative ultracentrifuge. Clin Chem 18, 499-502.

36. Hernández-Presa MA, Ortego M, Tuñón J, et al. (2003) Simvastatin reduces NF-kappaB activity in peripheral mononuclear and in plaque cells of rabbit atheroma more markedly than lipid lowering diet. Cardiovasc Res 57, 168-177.

37. Marin C, Ramirez R, Delgado-Lista J, et al. (2011) Mediterranean diet reduces endothelial damage and improves the regenerative capacity of endothelium. Am J Clin Nutr 93, 267-274.

38. Ross R (1999) Atherosclerosis is an inflammatory disease. Am Heart J 138, 419-420. 
39. Kutuk O \& Basaga H (2003) Inflammation meets oxidation: $\mathrm{NF}-\kappa \mathrm{B}$ as a mediator of initial lesion development in atherosclerosis. Trends Mol Med 9, 549-557.

40. Lee Y, Allport V, Sykes A, et al. (2003) The effects of labour and of interleukin 1 beta upon the expression of nuclear factor kappa B related proteins in human amnion. Mol Hum Reprod 9, 213-218.

41. Perez-Jimenez F, Ruano J, Perez-Martinez P, et al. (2007) The influence of olive oil on human health: not a question of fat alone. Mol Nutr Food Res 51, 1199-1208.

42. Bogani P, Galli C, Villa M, et al. (2007) Postprandial antiinflammatory and antioxidant effects of extra virgin olive oil. Atherosclerosis 190, 181-186.

43. Visioli F \& Bernardini E (2011) Extra virgin olive oil's polyphenols: biological activities. Curr Pharm Des 17 , 786-804.

44. Kreeft AJ, Moen CJ, Porter G, et al. (2005) Genomic analysis of the response of mouse models to high-fat feeding shows a major role of nuclear receptors in the simultaneous regulation of lipid and inflammatory genes. Atherosclerosis 182, 249-257.

45. Dell'Agli M, Fagnani R, Galli GV, et al. (2010) Olive oil phenols modulate the expression of metalloproteinase 9 in THP- 1 cells by acting on nuclear factor-kappaB signaling. J Agric Food Chem 58, 2246-2252.

46. Carluccio MA, Siculella L, Ancora MA, et al. (2003) Olive oil and red wine antioxidant polyphenols inhibit endothelial activation: antiatherogenic properties of Mediterranean diet phytochemicals. Arterioscler Thromb Vasc Biol $\mathbf{2 3}$ 622-629.

47. Llorente-Cortes V, Estruch R, Mena MP, et al. (2010) Effect of Mediterranean diet on the expression of pro-atherogenic genes in a population at high cardiovascular risk. Atherosclerosis 208, 442-450.

48. Mena MP, Sacanella E, Vazquez-Agell M, et al. (2009) Inhibition of circulating immune cell activation: a molecular antiinflammatory effect of the Mediterranean diet. Am J Clin Nutr 89, 248-256.

49. Niu J \& Kolattukudy PE (2009) Role of MCP-1 in cardiovascular disease: molecular mechanisms and clinical implications. Clin Sci 117, 95-109.

50. Grimble RF (1996) Interaction between nutrients, proinflammatory cytokines and inflammation. Clin Sci 91, 121-130.

51. Badimon JJ, Ibanez B \& Cimmino G (2009) Genesis and dynamics of atherosclerotic lesions: implications for early detection. Cerebrovasc Dis 27, 38-47.

52. Corti R, Farkouh ME \& Badimon JJ (2002) The vulnerable plaque and acute coronary syndromes. Am J Med 113, 668-680.

53. Hashim YZ, Eng M, Gill CI, et al. (2005) Components of olive oil and chemoprevention of colorectal cancer. Nutr Rev $\mathbf{6 3}$, 374-386.

54. Adhami VM, Ahmad N \& Mukhtar H (2003) Molecular targets for green tea in prostate cancer prevention. $J$ Nutr $\mathbf{1 3 3}$, S2417-S2424

55. Annabi B, Lachambre M, Bousquet-Gagnon N, et al. (2002) Green tea polyphenol (2)-epigallocatechin 3-gallate inhibits MMP-2 secretion and MT1-MMP-driven migration in glioblastoma cells. Biochim Biophys Acta 1542, 209-220.

56. Futterman LG \& Lemberg L (2002) High-sensitivity C-reactive protein is the most effective prognostic measurement of acute coronary events. Am J Crit Care 11, 482-486.

57. Kishimoto $T$ (2005) Interleukin-6: from basic science to medicine -40 years in immunology. Annu Rev Immunol 23, $1-20$.

58. Stow JL, Ching-Low P, Offenhäuser C, et al. (2009) Cytokine secretion in macrophages and other cells: Pathways and mediators. Immunobiology 214, 601-612.

59. Horvathova M, Jahnova E, Szabova M, et al. (2009) The relationship between cell surface markers, cytokines, ageing, and cigarette smoking. Bratisl Lek Listy 110, $394-400$. 